\title{
Violencia en el noviazgo: perpetración, victimización y violencia mutua. Una revisión
}

\author{
Dating violence: perpetration, and mutual violence. A review
}

\author{
Manoella Alegría del Ángel ${ }^{1}$ \\ Adriana Rodríguez Barraza ${ }^{2}$ \\ Universidad Veracruzana, México
}

\begin{abstract}
Resumen. Existen investigaciones cuyos resultados muestran tasas similares de violencia entre hombres y mujeres, incluyendo la violencia mutua, las cuales se contraponen a las teorías que hacen referencia a roles tradicionales de género. El objetivo fue realizar una revisión teórica acerca de los principales hallazgos de la violencia en el noviazgo en países latinos y de Norteamérica desde una perspectiva inclusiva de género. Se incluyeron estudios del año 2000 al 2013, cuya búsqueda se realizó en bases de datos. Se concluyó que los cambios socioculturales obligan a llevar a cabo un abordaje distinto de la violencia en jóvenes, considerando una visión inclusiva para promover relaciones de no violencia. Se recomienda profundizar en el papel de los roles de género.
\end{abstract}

Palabras clave. Violencia en el noviazgo, perpetración, victimización, violencia mutua, jóvenes, estudiantes.

Abstract. There are investigations whose results show similar rates of violence between men and women, including mutual violence, which are opposing to theories that refer to traditional gender roles. The aim of this study was to perform a theoretical review about the main findings of dating violence in Latin countries and North America from a gender inclusive perspective. Studies since 2000 to 2013 were included, the search was performed in databases. It was concluded that sociocultural changes require performing a different approach to violence in young, considering an inclusive vision to promote relationships of nonviolence. It is recommended to deepen in the paper of gender roles.

Keywords. Dating violence, young people, students, perpetration, victimization, mutual violence.

\footnotetext{
${ }^{1}$ Manoella Alegría del Ángel. Universidad Veracruzana. Dirección Postal: Av. Dr. Luis Castelazo Ayala s/n. Col. Industrial Ánimas, C.P. 91190 Xalapa, Veracruzana, México. E-mail: meni12346@hotmail.com.

${ }^{2}$ Adriana Rodríguez Barraza. Universidad Veracruzana. E-mail: arbarraza@hotmail.com
}

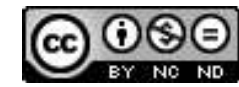




\section{Introducción}

De acuerdo con Armendáriz (2002), ha sido una creencia socialmente aceptada que el noviazgo es la época "ideal" de una pareja, sin embargo, la realidad se muestra diferente porque las jóvenes parejas muchas veces se enfrentan a situaciones violentas (Barilari, 2009). Este tema ha significado un verdadero reto conceptual y metodológico, dependiendo de la perspectiva teórica o ideológica adoptada para su elucidación, prevalecerán factores diversos (RojasSolís, 2011a).

Por ello, en el presente artículo se aborda la violencia en el noviazgo desde la Psicología de Género, considerando factores psicológicos, sociales y culturales que influyen en su construcción en ambos sexos (Barberá, 1998), es decir, junto a una concepción inclusiva de género que habla de la simetría de género (Hamel, 2007, 2009; Rojas-Solis, 2011a, 2013a). Es importante retomar tales enfoques, puesto que se han dedicado demasiados recursos al estudio de la violencia contra las mujeres, excluyendo al sexo opuesto (Álvarez, 2012). En este sentido, si bien es cierto que en las relaciones heterosexuales hay oportunidad de manifestar dominio, para ejercerlo es necesario que el receptor también sea un sujeto activo, que responda, reaccione y resista (Parada,1993; Rivera, Díaz-Loving, \& García, 2008). De acuerdo con González, Muñoz y Graña (2003), hay estudios que determinan un patrón de violencia en la que ambos miembros de la pareja emplean conductas agresivas como modalidad vincular, fungiendo así, un mismo individuo el papel de víctima y victimario.

Aunque en diversos países las relaciones de pareja siguen estando normadas por tradiciones culturales, en los jóvenes ha disminuido la asimetría de poderes, lo cual, revela cambios profundos en relación con el género y abriría caminos hacia la equidad (Amurrio, Larrinaga, Usategui \& Del Valle, 2010; Ibaceta, 2011; Rivera, et. al., 2008; Rojas-Solís, 2011a, 2013abc; Saldívar, Ramos, \& Romero, 2008; Straus, 2006). Lamentablemente dichos cambios no terminan de concretarse, pues resultan en interacciones violentas.
Por tal motivo, el objetivo de este estudio fue realizar una revisión teórica acerca de los principales hallazgos de la violencia en el noviazgo en países norteamericanos y latinos, con la finalidad de facilitar la reflexión sobre la importancia de emplear herramientas metodológicas que no omitan la posibilidad de una dinámica bidireccional de la violencia en parejas jóvenes. Se realizó una búsqueda de investigaciones sobre violencia en el noviazgo realizadas del año 2000 a 2013 en diversos países (Chile, Perú, Colombia, Puerto Rico, México, España, Estados Unidos de América, Canadá, Cuba), cuya búsqueda se realizó en diferentes bases de datos: Google Académico, Redalyc, Scielo, EBSCOhost, ELSEVIER, PubMed, SAGE y Dialnet.

No se usaron protocolos estrictos para evaluar el rigor metodológico con el fin de abarcar estudios con diferentes muestras o metodologías, sin embargo, se tomaron en cuenta algunos criterios de inclusión: muestras formadas por hombres y mujeres preuniversitarios y universitarios, y que tuvieran en cuenta la violencia ejercida o sufrida por ambos miembros de la pareja heterosexual (Ver Anexo 1). Cabe aclarar que se ha elegido dicho marco temporal y criterios de inclusión dada la importancia actual de la temática, así como la delimitación de la búsqueda de información.

\section{Desarrollo}

\section{Violencia en el noviazgo}

La palabra violencia proviene del latín violare, que significa infligir, quebrantar o abusar de otra persona (Romero, 2007), comprende un conjunto complejo de comportamientos, actitudes, sentimientos, prácticas, vivencias y estilos de relación entre los miembros de una pareja que producen daños, malestar y pérdidas personales (Pueyo, 2009). La violencia en el noviazgo hace referencia al uso o amenaza de la fuerza física, restricción, abuso psicológico y/o sexual dañando o causando dolor a la pareja (Morales \& Rodríguez, 2012).

Póo y Vizcarra (2008) señalan características referentes a la dinámica de la violencia: escalada (incremento de las conductas violentas), dirección (referida a quién ejerce y quién recibe violencia), traspaso de límites (trasgresión de 
acuerdos implícitos y explícitos establecidos por la pareja) y expresión según género (forma que adopta la conducta violenta de acuerdo al género de quien las ejerce). Así, la violencia suele situarse en las relaciones de forma gradual, teniendo un pronóstico nada favorable para los novios porque una vez puesta en marcha, tiende a continuar y a agravarse (González-Ortega, Echeburúa, \& Corral, 2008).

\section{Clasificación de la violencia}

En función de la naturaleza de los actos, el daño ocasionado o los medios empleados, generalmente es clasificada en física, psicológica y sexual (Sánchez, 2009; Romero, 2007). La física comprende cualquier acto, no accidental, que provoque o pueda producir daño a la integridad física de un individuo como lesiones leves o graves (López, 2004; Romero, 2007; Ruiz, 2007). Es el tipo más fácilmente detectado (Espinoza \& Pérez, 2008) y los medios para ejercerla implican el sometimiento corporal mediante instrumentos o el propio cuerpo (Romero, 2007). De acuerdo con Póo y Vizcarra (2008) los universitarios expresan este tipo de violencia mediante conductas como golpear, apretar, empujar, pellizcar, tirar el pelo y patear. En cuanto a las tasas de la violencia física grave los porcentajes más bajos son para el uso de armas, y los más altos para empujones e intento de estrangulamiento (Castellano, García, Lago, \& Ramírez, 1999).

La psicológica implica acción u omisión, se hiere a otra persona sin dejar huellas visibles (Espinoza \& Pérez, 2008; Ruiz, 2008). Produce un daño en la esfera emocional, el medio fundamental que se utiliza es la comunicación verbal (Pueyo, 2009; Romero, 2007). Además, se presenta en parejas jóvenes mediante comportamientos como exigir, criticar, manipular, controlar, humillar, insultar, no respetar acuerdos, ignorar, descalificar y discriminar (Castellano, et al., 1999; Ferrer, 2009; Póo \& Vizcarra, 2008; Rojas-Solís, 2013c).

Finalmente, la violencia sexual se define como cualquier tipo de presión física o emocional ejercida por una persona para imponer a otra actos de orden sexual (Romero, 2007; Ruíz, 2007; Saldívar, et al., 2008). Se caracteriza por acciones de sexo forzado, hostigamiento sexual, humillación sexual, inducción a la pornografía o prostitución, tocamientos lascivos o la violación (López, 2004; Pueyo, 2009; Romero, 2007; Sánchez, 2009).

\section{Prevalencia}

El maltrato psicológico suele ser el más reportado en población joven (Cortaza, Mazadiego \& Ruíz, 2011; Instituto Mexicano de la Juventud, 2008; Rey-Anacona, Mateus-Cubides \& Bayona-Arévalo, 2010; Villafañe, Jiménez, Carrasquillo \& Vázquez, 2012; Vizcarra \& Póo, 2011). El $45.3 \%$ de las mujeres y el $71.9 \%$ de los hombres manifestó que en sus relaciones había violencia de este tipo (Mañas, Martínez, Esquembre, Montesios \& Gilar, 2012). Tasas similares de hombres $(17 \%)$ y mujeres $(16 \%)$ informaron de algún tipo de violencia en los últimos seis meses (Saewyc et al., 2009). Al mismo tiempo, Wekerle, et al. (2009) encontraron que la violencia era común en más de la mitad de las mujeres (63-67\%) y casi la mitad de los hombres (4449\%). Moral y López (2013b) hallaron que la media de violencia ejercida fue mayor que la recibida, y las mujeres reportaron recibir menos violencia que los hombres, pero sus medias de violencia ejercida fueron equivalentes a las de ellos.

\section{Perpetración}

La perpetración hace referencia a quién ejecuta el acto violento, algunos estudios afirman que las mujeres pueden iniciar interacciones agresivas físicamente con mayor frecuencia que sus parejas masculinas (Dasgupta, 2002). Murray, Wester y Paladino (2008) hallaron que de los universitarios con violencia en el noviazgo, 73\% la habían perpetrado contra sus parejas en el último mes. $\mathrm{Al}$ mismo tiempo, Swahn et. al. (2008) identificaron que el $24.8 \%$ de los integrantes de la relación de pareja cumple el papel de victimario. Otro trabajo mostró que $37 \%$ de las mujeres y $23 \%$ de los hombres ejercieron violencia hacia su pareja (Straus, et al., 2009).

De acuerdo con Villafañe, et. al. (2012), las experiencias más reportadas por los estudiantes fueron haber gritado o insultado a su pareja (54\%), haberla criticado o humillado $(39 \%)$, haber destruido objetos o golpear la pared por enojo $(34 \%)$ y presentar conducta controladora (34\%). Las mujeres fueron más propensas a arrojar objetos, 
abofetear, patear y morder; los hombres fueron más propensos a dar palizas, asfixiar o estrangular (Archer, 2002). Por medio de una investigación en estudiantes universitarios, se dio a conocer que las mujeres ejercen violencia por medio de callar al otro fuertemente, gritos, arañazos, bofetadas y patadas, y los hombres, recurren a los chupetones ("hematomas causados por una fuerte succión con la boca"), quemaduras con cigarro, cerillo o encendedor, intento de estrangulamiento y amenaza con arma de fuego (Oliva, González, Yedra, \& Rivera, 2012).

Según Muñoz-Rivas, Graña, O’Leary y González (2009), el porcentaje de hombres agresores es superior al de mujeres $(35.7 \%$ vs $14.9 \%)$. Otros resultados arrojan que el $35 \%$ de los varones había presentado violencia psicológica, $15 \%$ física y el 17\% sexual; en comparación, el $47 \%$ de las chicas informaron violencia psicológica, $28 \%$ física y el $5 \%$ sexual (Sears, Byers, \& Price, 2007).

Straus y Gozjolko (2007) encontraron que $24 \%$ de los varones y $31.2 \%$ de las mujeres atacaron físicamente a su pareja, donde ellas presentaron el doble de probabilidades que ellos de describirse como la única persona violenta $(20.7 \%$ vs $9.4 \%)$. En el estudio de Giordano, Soto, Manning y Longmore (2010), el 18.3\% de estudiantes perpetraron actos de violencia contra su pareja, encontrándose un mayor número de mujeres $(34.9 \%)$ que de hombres (6.2\%). Otros autores demostraron puntajes más altos en las agresiones verbales-emocionales cometidas por ellas (Rojas-Solís, 2011; Rojas-Solís \& Carpintero, 2011). También, Muñoz-Rivas, Andreu, Graña, O'Leary y González (2007) encontraron que las mujeres adolescentes tienden a manifestar un mayor uso de tácticas agresivas psicológicas y físicas leves que los hombres. Asimismo, Muñoz-Rivas, Graña y O’Leary (2007) revelan que un porcentaje mayor de mujeres ejercen violencia verbal ( $95.3 \%$ vs $92.8 \%$ ), mientras que los hombres se dedican a más a la violencia física grave $(4.6 \%$ vs $2 \%)$.

Rivera-Rivera, Allen-Leigh, Rodríguez-Ortega, ChávezAyala y Lazcano-Ponce (2007), hallaron que las mujeres son quienes perpetran mayormente la violencia física y los hombres la psicológica. Igualmente se encontraron tasas más altas de perpetración de la violencia física en mujeres que en hombres (Corral, 2009; Dasgupta, 2002;
Desmarais, Reeves, Nicholls, Telford y Fiebert, 2012b; Muñoz-Rivas, et al., 2007; O'Leary, Smith, Avery-Leaf, \& Cascardi, 2008). Para Archer (2000), las mujeres tenían una probabilidad ligeramente mayor que los hombres a utilizar uno o más actos de agresión física. Por otro lado, Álvarez (2012) halló más estudios con un registro mayor de perpetración de violencia física o iniciación de las agresiones por las mujeres que por los hombres. Mientras más de una de cada cuatro mujeres ejerce violencia física contra su pareja, uno de cada cinco hombres informa haberla perpetrado (Desmarais et al., 2012b).

Rey-Anacona, Mateus-Cubides y Bayona-Arévalo (2010), encontraron que el $87.9 \%$ de los participantes ejercieron maltrato, descubriéndose que el número de varones que ejerció violencia de tipo emocional y sexual fue significativamente mayor al de mujeres. Según Vara y Roa (2000), el $88.3 \%$ de los varones y el $93.2 \%$ de las mujeres atacaron psicológicamente a sus parejas, un mayor porcentaje de varones las atacaron físicamente $(54.8 \%$ vs $48.8 \%$ ), finalmente, el $16 \%$ de varones y el $7.3 \%$ de mujeres informaron haberlas coercionado sexualmente.

Con base en el estudio de Rey-Anacona (2013), el $85.6 \%$ de jóvenes reportaron haber ejercido por lo menos una conducta violenta, se encontró que el porcentaje de varones que informó maltrato emocional (43.9\% vs $32.2 \%)$, sexual ( $29 \%$ vs $17.5 \%$ ), económico $(15.1 \%$ vs $7.8 \%)$ y negligente $(20.4 \%$ vs $10.1 \%)$ fue significativamente mayor que el de mujeres, no hallándose diferencias para los tipos físico y psicológico. Gámez, Straus y Hershberger (2011) hicieron un análisis en 32 países (África subsahariana, Asia, Europa, América Latina, Oriente Medio, América del Norte y Oceanía), encontrando que el $26.7 \%$ de los varones y el $19.6 \%$ de las mujeres habían forzado a su pareja a tener relaciones sexuales. Otros estudios encuentran que hay un mayor reporte de hombres que ejercen violencia sexual (Corral, 2009; Graña, Rodríguez, \& Peña, 2009; Rojas-Solís \& Carpintero, 2011).

Es innegable que las mujeres son capaces de ejercer violencia contra sus parejas, misma que es comparable a la de los hombres en términos de contexto, motivación, resultados y consecuencias (Dasgupta, 2002). No obstante, es cierto que la violencia física perpetrada por 
las mujeres es menos probable que resulte en lesión, y que las mujeres sufren mayores tasas de lesiones graves (Archer, 2000; Chiodo, et al., 2011; Dasgupta, 2002; Desmarais, et al., 2012b; Muñoz-Rivas, Graña, \& O'Leary, 2007).

\section{Victimización}

La victimización hace referencia a quién recibe los actos violentos. En el caso de los varones, se reportan actos de victimización por medio de amenazas, empujones, cachetadas y patadas, mientras que las mujeres tienden a ser víctimas de insultos, indiferencia, amenazas, golpes con objetos, empujones, cachetadas y patadas (O'Leary, Smith, et al., 2008). Tomando en cuenta a Swahn, et al. (2008), el 30.7\% de los integrantes de una relación de noviazgo sufre de victimización, para Giordano, et. al. (2010), el 32.7\% lo hace (46\% mujeres y $13.2 \%$ hombres). También, Murray, et al. (2008) descubrieron que el 48\% de los universitarios la habían experimentado. De acuerdo con Villafañe, et al. (2012), las experiencias de violencia más reportadas fueron críticas o humillaciones (48\%), gritos e insultos (47\%) y conducta controladora (46\%).

En otro estudio el $25.7 \%$ de las mujeres informaron que sus parejas masculinas las habían violentado, por otro lado, los varones fueron dos veces más propensos que las mujeres a indicar sólo victimización (13.7\% frente a $6.7 \%$ ) (Straus \& Gozjolko, 2007). En cuanto a dicha victimización sufrida por los varones existen estudios que demuestran que reciben mayor violencia psicológica (Comezaña, 2006; Lehrer, et al., 2009; O'Leary, et al., 2008; Saewyc, et al., 2009) y física (Comezaña, 2006; Lehrer, et al., 2009; O’Leary et al., 2008; Rivera-Rivera, et al., 2007; Rojas-Solís, 2011; Rojas-Solís \& Carpintero, 2001).

Dasgupta (2002) encontró que más varones (31\%) reportaron que sus parejas los habían agredido físicamente. Por su parte, Lehrer, et al., (2009) observaron que el $15.1 \%$ de las mujeres y el $26.6 \%$ de los hombres reportaron alguna forma de victimización física, y respecto a las lesiones físicas, el $15.9 \%$ de las mujeres y el 6.9\% de los hombres informaron al menos una lesión. Chiodo et al. (2011) y Comezaña (2006) coinciden en que las mujeres reciben mayor daño físico en comparación con los varones. Aunado a ello, Álvarez (2012) descubrió que los hombres sufren mayores niveles de victimización por violencia física. Además, en otra revisión se concluyó que aproximadamente una de cada cuatro mujeres y uno de cada cinco hombres experimentaron este tipo de violencia en su relación (Desmarais, et al., 2012a).

Por el contrario, existe evidencia que prueba que la victimización de la violencia psicológica es más sufrida por las mujeres (Rivera-Rivera et al., 2007). Muñoz-Rivas, et al. (2007) observaron que el porcentaje de mujeres que manifestaron ser víctimas fue significativamente mayor que de hombres para el caso de críticas, golpes, patadas, empujones o haber sido sujetadas, y el porcentaje de hombres víctimas fue mayor en el caso de amenazas y cachetadas. Las mujeres reportan mayor porcentaje de victimización para violencia sexual (Graña, Rodríguez \& Peña, 2009; Muñoz-Rivas, Graña, O’Leary \& González, 2009; Saewyc et al., 2009; Straus, et al., 2009; Vara \& Roa, 2000).

En México, según la Encuesta Nacional de Violencia en las Relaciones de Noviazgo levantada en contexto rural y urbano, el 15\% de los jóvenes habían experimentado violencia física (61.4\% mujeres y $46 \%$ hombres); $76 \%$ de los jóvenes fueron víctimas de violencia psicológica, y el $16.4 \%$ de las chicas declararon violencia sexual (Instituto Mexicano de la Juventud, 2008). A partir de otro estudio, se encontró que $45.5 \%$ de hombres y $46.8 \%$ de mujeres reportaron sufrir violencia, no encontrándose diferencias significativas entre géneros (Peña, et al., 2013).

Como se observa, existe un cuerpo creciente de investigaciones que examinan las experiencias de las victimas masculinas de la violencia de pareja, y demuestra que los hombres tienen secuelas como resultado de la victimización (Desmarais, et al., 2012a). Las investigaciones sobre perpetración femenina han ido en aumento, en contraste, los estudios de victimización masculina son menos; ello puede tener importantes implicaciones, ya que si bien la noción de que las mujeres usan la violencia contra sus parejas está ganando aceptación, las experiencias de los varones continúan siendo descuidadas (Desmarais, et al., 2012b). 


\section{Violencia mutua}

Hay otro grupo de investigaciones donde se aborda la simetría de género dejando atrás el modelo unidireccional y haciendo alusión a la violencia mutua, situación en la que los miembros de una pareja se atacan física, sexual o psicológicamente de manera recíproca (Giordano, et al., 2010; Ibaceta, 2011; Johnson, 2006; O'Leary, et al., 2008; Salazar \& Vinet, 2011). De hecho, esta simetría ha desafiado la teoría prevaleciente acerca de la violencia de pareja que habla de una asimetría de género (Desmarais, et al., 2012b), debido a que, los detractores de la teoría feminista reclaman que hombres y mujeres la utilizan por igual (Dasgupta, 2002). Existen diversas investigaciones que demuestran la existencia de esta forma de violencia en las parejas jóvenes (Chiodo et al., 2011; Comezaña, 2006; Giordano, et al., 2010; Leal, Reinoso, Rojas, \& Romero, 2011; Medeiros \& Straus, 2006; O'Leary, et al., 2008; Straus, 2006; Straus \& Gozjolko, 2007; Straus \& Mickey, 2012). De acuerdo con Giordano, et al. (2010), el 49\% de los estudiantes reportaron violencia mutua (46.9\% hombres y $51.9 \%$ mujeres).

Según O’Leary, Smith, Avery-Leaf y Cascardi (2008), aproximadamente el $94 \%$ de los hombres y las mujeres informaron que la violencia psicológica era mutua. También, Corral (2009) encontró que el mayor porcentaje es para las relaciones donde ambos miembros han abusado psicológicamente de sus parejas (90.3\%), al mismo tiempo, el $62.7 \%$ de los hombres y $48.3 \%$ de las mujeres declararon hallarse en una relación violenta físicamente donde ambos miembros la utilizaban. En otra investigación el patrón más frecuente fue la violencia mutua, el $62.8 \%$ de los universitarios reportaron violencia física, el 93.1\% psicológica y el 64.4\% sexual (Straus \& Douglas, 2004). Más hallazgos demostraron que hombres y mujeres tuvieron violencia mutua tanto física como psicológica (Arias, Azbell \& Valencia, 2010; FernándezFuertes \& Fuertes, 2010; Leal, et al., 2011).

Otros datos que alertan sobre la existencia de patrones de violencia mutua son las correlaciones encontradas entre cometer una determinada agresión y ser víctima de esa misma forma de violencia, sobre todo en la violencia sexual y las agresiones verbalesemocionales (Fernández-Fuertes, Fuertes, \& Pulido,
2006). De acuerdo con la investigación de RojasSolís (2011b) no hubo diferencias significativas entre hombres y mujeres en cuanto a las agresiones sexuales sufridas, lo cual podría estar apoyando la idea de un "doble rol" y su posible bidireccionalidad. En un estudio de casos, Romero (2007) encontró que la primera pareja expresaba el poder de forma bidireccional, y en la segunda aunque el poder era mutuo, hubo más experiencias en las que el hombre ocupó la posición de víctima.

Más resultados muestran un patrón recíproco en la agresión psicológica, pero a medida que se agrava la expresión conductual de la violencia, se incrementa su unidireccionalidad (Rubio-Garay, López-González, Ángel, \& Sánchez-Elvira-Paniagua, 2012). Al comparar parejas de novios con parejas casadas o que cohabitan, en las segundas, hay menos violencia mutua (Straus, 2006). En este sentido, la violencia en el noviazgo no es un fenómeno que toma sólo una forma determinada, hay situaciones que siguen una pauta unidireccional y hay otras en donde la pauta se asemeja más a la simetría (Ibaceta, 2011; Rojas-Solís, 2013a, 2013b).

Hasta el momento se han señalado investigaciones acerca de la violencia en el noviazgo en cuestión de victimización, perpetración y violencia mutua, cuyos resultados sonvariados eincluso se contraponen alas teorías que hacen referencia a los roles tradicionales de género. Desde la perspectiva feminista, la violencia en parejas heterosexuales es el resultado de la sociedad patriarcal en donde el varón ha tenido el papel predominante, por lo que propone un modelo unidireccional de su medición, que consiste en estudiar únicamente los actos ejercidos por hombres contra mujeres (Hamel, 2007, 2009; RojasSolís, 2011b). Sin embargo, existe evidencia como la aquí presentada, de que las mujeres son al menos tan violentas como los hombres (Espinoza \& Pérez, 2008; Johnson, 2006), enfatizándose la simetría de género.

Al concluirse que la violencia se caracteriza por ser simétrica, los fundamentos teóricos del paradigma patriarcal y la teoría feminista empiezan a cuestionarse a partir de una perspectiva inclusiva de género (Álvarez, 2012; Dasgupta, 2002; Desmarais, et al., 2012b; Esquivel-Santoveña, 2012; Hamel, 2007, 2009; Ibaceta, 
2011; Rojas-Solís, 2011a, 2013a, 2013b). Desde tal concepción, se considera un modelo bidireccional de la violencia que aplica instrumentos a hombres y mujeres por igual, arrojando muchas veces en sus conclusiones niveles similares de agresiones cometidas y sufridas para ambos sexos, haciendo hincapié en la simetría de género y la violencia mutua (Hamel, 2009, Rojas-Solís, 2013c).

Ello alentado por los cambios socioculturales como el acceso de las mujeres a la educación, su participación en el trabajo asalariado y la mayor participación del hombre en las tareas domésticas y en la crianza de los hijos (Moral \& López, 2013a; Orozco, Nievar, \& Middlemiss, 2012), generándose actitudes cada vez más liberales hacia los roles de género (Sears, et al., 2007) y menos afines a los estereotipos tradicionales (Orozco, et al., 2012; Straus, 2006).

Un estudio que concede una posible explicación a tales cambios expone que gran parte de las familias de las que provienen los jóvenes se encuentran en transición a relaciones más igualitarias (es decir, menos tradicionalistas), donde las normas de convivencia remiten a una autoridad compartida por ambos miembros de la pareja, y el 80\% de los jóvenes rechazan que, para el buen funcionamiento de la relación, la mujer haya de ser sumisa y, por el contrario, comparten la convicción de que ser el único proveedor no otorga el poder en el hogar, así como, la idea de que las mujeres no están obligadas a satisfacer sexualmente a su pareja (Amurrio, et al., 2010).

Straus (2006), concuerda en que existe una disminución de la estructura tradicional; al analizar el dominio que se ejerce en la pareja, Tanzania, Rusia, Irán, Taiwán y China continental fueron los países con mayor dominación masculina, por el contrario, Suecia, Países Bajos, Canadá, Suiza y Malta presentaron los puntajes más bajos, ya que, han promovido la igualdad de género y presentan un mayor desarrollo económico y modernidad. No obstante, se ha encontrado que el dominio ya sea por la pareja masculina (Straus, 2006; Straus \& Mickey, 2012) o femenina está fuertemente asociado con una mayor probabilidad de que ambos miembros de la pareja sean violentos (Straus, 2006).

\section{Conclusiones}

Sin duda, en la violencia de pareja siempre hay una víctima y un victimario que, en incontables estudios, se han estado presentando como una dicotomía permanente con un hombre activo y una mujer pasiva, sin considerar que la violencia es un fenómeno humano y relacional en movimiento, y no sólo uno de género, por lo que no se puede reducir fácilmente en dicotomías rígidas (Hamel 2007, 2009; Rojas-Solís, 2011a).

Los resultados aquí revisados son contundentes pues revelan datos que aluden al reconocimiento de que esta problemática no corre con la misma suerte que en años anteriores, debido a que las mujeres están adoptando también el papel de victimarias (Romero, 2007). En este orden de ideas, se hablaría de la ruptura del paradigma tradicional de género, principalmente en relación a la sumisión de las mujeres que, en última instancia, dan cobertura a la violencia, aumentando considerablemente las exigencias de las jóvenes en el seno de la relación y desvinculándolas de los roles tradicionales (Amurrrio et al., 2010; Moral \& López, 2013; Orozco, et. al., 2012; Sears, et al., 2007; Straus, 2006).

Por lo tanto, aunado a que es innegable el incremento de las investigaciones acerca del fenómeno de la violencia en parejas jóvenes, también lo es el hecho de que, tanto a nivel de la pareja como de la sociedad en general, se están presentando cambios socioculturales inminentes que obligan a presentar un abordaje distinto, considerando una perspectiva inclusiva de género y un modelo bidireccional de la violencia (Álvarez, 2012; Esquivel-Santoveña, 2012; Hamel, 2007, 2009; RojasSolís, 2011a, 2013a, 2013b). Y a partir de dicho modelo asumir que la víctima, sea hombre o mujer, también es capaz de llevar a cabo acciones para enfrentar dicha violencia activamente y el victimario también podría asumir la pasividad (Rojas-Solís, 2011a).

De tal modo que, independientemente del género, lass personas jóvenes son susceptibles de recibir y al mismo tiempo ejercer violencia en su noviazgo (Arias, et al., 2010; Chiodo et al., 2011; Comezaña, 2006; Corral, 2009; Fernández-Fuertes \& Fuertes, 2010; Giordano, et al., 2010; Leal, et al., 2011; Medeiros \& 
Straus, 2006; O’Leary, et al. 2008; Rojas-Solís, 2011b; Straus, 2006; Straus \& Douglas, 2004; Straus \& Gozjolko, 2007), por lo cual, la simetría de género y la violencia mutua tendrían que empezar a formar parte del lenguaje coloquial, por lo menos cuando se hable de adolescentes y jóvenes adultos; así como, tomar en cuenta la importancia de hacer una distinción entre los tipos de violencia, puesto que no pueden tener las mismas causas, la trayectoria del desarrollo, las consecuencias, o el pronóstico para una intervención efectiva (Ibaceta, 2011; Johnson, 2006).

Se sugiere que países que promueven la igualdad de género y presentan un mayor desarrollo económico y modernidad, han logrado disminuir la estructura tradicional (Amurrrio, et al., 2010; Straus, 2006), por tal motivo, es indispensable considerar acciones encauzadas hacia el mismo sentido, sin incurrir en la potencialización de la mutualidad de las agresiones, es decir, que la simetría de género no sólo se vea reflejada en la posibilidad de cada hombre y mujer de producir dichos actos, sino que se extienda a otros contextos en términos efectivos para la sociedad. Para ello, es necesario sumar esfuerzos y generar estrategias que promuevan cambios sociales positivos, pero sobre todo, intervenciones específicas a nivel individual y relacional, que tomen en cuenta las particularidades de la relación, permitiendo la disminución de la incidencia y la cronicidad de la violencia en las relaciones de noviazgo.

Entre las limitaciones de esta revisión fueron la desproporción de las muestras, así como el acceso restringido a muchos de los estudios existentes, sin embargo, se aspiró a la sistematización considerando los criterios de inclusión mencionados con anterioridad. La mayoría de los estudios cuentan con un diseño transversal y cuantitativo, por lo que deben considerarse otro tipo de metodologías como la cualitativa y el diseño comparativo y longitudinal, haciendo análisis por grupos etarios y profundizando en las premisas socioculturales que inciden en la violencia y enmarcan los cambios de la dinámica relacional. Se recomienda considerar la inclusión de género para analizar el papel de los roles y estereotipos de género en la violencia en el noviazgo.
Finalmente, el principal aporte de esta revisión fue marcar una visión distinta del abordaje de la violencia, sin exclusividad de sexo, considerando la simetría de género, la edad y los instrumentos de medición utilizados, así como fortaleciendo la creciente línea de investigación sobre la reciprocidad de los actos violentos en el noviazgo. En la medida en que esto se tome en cuenta, las estrategias de prevención e intervención que se formulen, a partir de los resultados encontrados, serán más especializadas y sólidas, obteniéndose beneficios en términos de salud y bienestar social.

\section{Referencias}

Alvarez, J. (2012). Análisis comparativo de una recopilación de estudios internacionales sobre la violencia en pareja. (Documento en sitio web). Recuperado de http:// www.escorrecto.org/400razones.pdf

Amurrrio, M., Larrinaga, A., Usategui, E., \& Del Valle, A. (2010). Violencia de género en las relaciones de pareja de adolescentes y jóvenes de Bilbao. Ekaina, 47, 121-134.

Archer, J. (2000). Sex Differences in Aggression Between Heterosexual Partners: A Meta-Analytic Review. Psychological Bulletin, 126(5), 651-680.

Archer, J. (2002). Sex differences in physically aggressive acts between heterosexual partners. A meta-analytic review. Aggression and Violent Behavior, 7, 313-351. doi: s1359-1789(01)00061-1

Arias, K.P., Azbell, E., \& Valencia, S. (2010). Violencia en parejas adolescentes. (Documento en sitio web) Recuperado de http://www.tlalpan.uvmnet. edu/oiid/download/Violencia \%20Parejas $\% 20$ Adolescentes_04_CSO_PSIC_PICSJ_E.pdf

Armendáriz, R. (2002). PNL: Ayudando a amar amando. México: Pax.

Barberá, E. (1998). Psicología del Género. Barcelona, España: Ariel.

Barilari, S. (2009). Hacia la prevención de noviazgos violentos. (Documento en sitio web) Recuperado de http:/ / www.ecapsocial.com.ar/files/Noviazgos_ Violentos.pdf 
Castellano, I.A., García, M., Lago, M., \& Ramírez, L. (1999). La violencia en las aprejas universitarias. Boletin criminológico, 42, 1-4.

Chiodo, D., Crooks, C., Wolfe, D., McIsaac, C., Hughes, R., \& Jaffe, P. (2011). Longitudinal prediction and concurrent functioning of adolescent girls demonstrating various profiles of dating violence and victimization. Prevention Science, 13(4), 350-359. doi: 10.1007/s11121-011-0236-3

Comezaña, K. (2006). Violencia y negociación en estudiantes enamorados con instrucción superior. (Documento en sitio web) Recuperado de http:/ / www.aristidesvara. net/pgnWeb/tesis/licenciatura/violencia_ negociacion/Articulo_tesis\%20licenciatura.pdf

Corral, S. (2009). Estudio de la violencia en el noviazgo en jóvenes universitarios/as: cronicidad, severidad y mutualidad de las conductas violentas. Psicopatología Clínica Legal y Forense, 9, 29-48.

Cortaza, L., Mazadiego, T., \& Ruíz, S. (2011). Prevalencia de violencia en el noviazgo en estudiantes preuniversitarias de Minatlitlán, México. Exploratoris, 2, 13-18.

Dasgupta, D. S. (2002). A framework for understanding women's use of nonlethal violence in intimate heterosexual relationships. Violence against women, 8(11), 1364-1389. doi: 10.1177/107780102237408

Desmarais, S., Reeves, K., Nicholls, T., Telford, R., \& Fiebert, M. (2012a). Prevalence of physical violence intimate relationships, Part 1: rates of male and female victimization. Partner Abuse, 3(2), 140-169. doi: 10.1891/1946-6560.3.2.e1

Desmarais, S., Reeves, K., Nicholls, T., Telford, R., \& Fiebert, M. (2012b). Prevalence of Physical Violence in intimate relationships, Part 2: rates of male and female perpetration. Partner Abuse, 3(2), 170-198. doi: http://dx.doi.org/10.1891/1946-6560.3.2.140

Díaz-Loving, R. (2008). De la psicología universal a las idiosincrasias del mexicano. En R. Díaz-Loving, Etnopsicología mexicana. siguiendo la buella teórica y empírica de Diaz-Guerrero (pp. 25-41). México: Trillas.
Espinoza, A. \& Pérez, G. (2008). Percepciones sociales acerca de los hombres víctimas de la violencia por parte de su pareja (Tesis de licenciatura). Universidad de Costa Rica. Recuperado de http://www.ts.ucr.ac.cr/binarios/ tfglic/tfg-1-2008-11.pdf

Esquivel-Santoveña, E.E. (2012). Investigating the rates, aetiology and consequences of physical and psychological intimate partner violence in international university students. (Tesis doctoral). College of Life and Environmental Sciences of the University of Birmingham.

Fernández-Fuertes, A. A., Fuertes, A. \& Pulido, R. F. (2006). Evaluación de la violencia en las relaciones de pareja de los adolescentes. Validación del Conflict in Adolescent Dating Relationships Inventory (CADRI) - versión española. International Journal of Clinical and Health Psychology, 6(2) 339-358.

Fernández-Fuertes, A., \& Fuertes, A. (2010). Physical and psychological aggression in dating relationships of Spanish adolescents: Motives and consequences. Child Abuse \& Neglect, 34, 183-191. doi:10.1016/j. chiabu.2010.01.002

Ferrer, D. (2009). Alternativa de intervención desde las competencias comunicativas para minimizar la violencia psicológica en parejas rurales y suburbanas. (Documento en sitio web) Recuperado de: http://tesis.repo.sld. cu/187/1/Ferrer_Lozano.pdf

Gámez, M., Straus, M., \& Hershberger, S. (2011). Childhood and Adolescent Victimization and Perpetration of Sexual Coercion by Male and Female University Students. Deviant Behavior, 1-39. doi: 10.1080/01639625.2010.514213

García, B., \& Bedolla, P. (1993). Las relaciones de poder y violencia vinculadas al hostigamiento sexual. En P. Bedolla, O. Bustos, G. Delgado, B. García, \& L. Parada, Estudios de género y feminismo II (pp. 1-432). D.F., México: Fontamara.

Giordano, P., Soto, D., Manning, W., \& Longmore, M. (2010). The characteristics of romantic relationships associated with teen dating violence. Social Science Research, 39, 863-874. doi:10.1016/j. ssresearch.2010.03.009 
González-Lozano, P., Muñoz, M. \& Graña, J. (2003). Violencia en las relaciones de pareja en adolescentes y jóvenes: una revisión. Psicopatología clínica Legal y Forense, 3(3), 23-39.

González-Ortega, I., Echeburúa, E., \& Corral, P. (2008). Variables significativas en las relaciones violentas en parejas jóvenes: una revisión. Psicología conductual, 16(2), 207-225.

Graña, J. L., Rodríguez, M. J., \& Peña, M. E. (2009). Agresión hacia la pareja en una muestra de la comunidad de Madrid: Análisis por Género. Psicopatología Clínica, Legaly Forense, 9, 7-28.

Hamel, J. (2007). Toward a Gender-Inclusive Conception of Intimate Partner Violence Research and Theory: Part 1 - Traditional Perspectives. International Journal of Men's Health, 6(1), 36-53. doi: 10.3149/jmh.0601.36

Hamel, J. (2009). Toward a Gender-Inclusive Conception of Intimate Partner Violence Research and Theory: Part 2 - New Directions. International Journal of Men's Health, 8(1), 41-59. doi: 10.3149/ jmh.0801.41

Ibaceta, F. (2011). Violencia en la pareja: ¿Es posible la terapia conjunta? Terapia Psicológica, 29(1), 117-125.

Instituto Mexicano de la Juventud. (2008). Encuesta Nacional de violencia en las relaciones de noviazgo 2007. México: SEP.

Johnson, M. (2006). Conflict and Control. Gender Symmetry and Asymmetry in Domestic Violence. Violence Against Women, 12(11), 1003-1018.

Leal, F., Reinoso, L., Rojas, K., \& Romero, R. (2011). Violencia en las relaciones de pareja en adolescentes escolares de Arica. Revista Infancia y Educación, 1(1), 18-35.

Lehrer, J. A., Lehrer, E. L., \& Zhenxiang, Z. (2009). Physical and psychological dating violence in young men and women in Chile: results from a 2005 survey of university students. International Journal of Injury Control \& Safety Promotion, 16(4), 205-214. doi: 10.1080/17457300903307003
López, E. (2004). La figura del agresor en la violencia de género: características personales e intervención. Papeles del Psicólogo, 25(88), 31-38.

Mañas, C., Martínez, A., Esquembre, M., Montesios, N., \& Gilar, R. (2012). Exploración de la violencia en las relaciones de pareja de jóvenes universitarias/os. Recuperado de http://web.ua.es/es/ice/jornadasredes-2012/documentos/posters/245605.pdf

Medeiros, R., \& Straus, M. (2006). Risk Factors for Physical Violence Between Dating Partners: Implications for Gender-Inclusive Prevention and Treatment of Family. En J. Hamel, \& T. Nicholls (Eds.). Family Approaches in domestic Violence: A Practitioner's Guide to Gender-Inclusive Research and Treatment: Springer. Recuperado de http://pubpages. unh.edu/ mas2/ID28-PR28.pdf

Moral, J., \& López, F. (2013a). Premisas socioculturales y violencia en la pareja: diferencias y semejanzas entre hombres y mujeres. Estudios sobre las Culturas Contemporáneas, XIX(38), 47-71.

Moral, J., \& López, F. (2013b). Violencia de pareja en personas que viven o no con su parea y en ambos sexos. Psicogente, 16(30), 296-310.

Muñoz-Rivas, M., Andreu, J. M., Graña, J. L., O’Leary, D., \& González, M. (2007). Validación de la versión modificada de la Conflicts Tactics Scale (M-CTS) en población juvenil española. Psicothema, 19(4), 693-698.

Muñoz-Rivas, M., Graña, J. L., \& O’Leary, D. (2007). Aggression in Adolescent Dating Relationships: Prevalence, Justification, and Health Consequences. Journal of Adolescent Health, 40, 298-304. doi:10.1016/j. jadohealth.2006.11.137

Muñoz-Rivas, M., Graña, J. L., O’Leary, D., \& González, M. (2009). Prevalence and predictors of sexual aggression in dating relationships of adolescents and young adults. Psicothema, 21(2), 234-240.

Murray, C. E., Wester, K. L., \& Paladino, D. A. (2008). Dating Violence and Self-Injury Among Undergraduate College Students: Attitudes and Experiences. Journal Of College Counseling, 11(1), 42-57. 
O'Leary, D., Smith, A., Avery-Leaf, S., \& Cascardi, M. (2008). Gender Differences in Dating Aggression Among Multiethnic High School Students. Journal of Adolescent Health, 42, 473-479. doi:10.1016/j. jadohealth.2007.09.012

Oliva, L., González, M., Yedra, L., \& Rivera, E. y. (2012). Agresión y manifestaciones violentas en el noviazgo en universitarios. Psicologia.com, 16(1), 1-13.

Orozco, A. E., Nievar, M. A., \& Middlemiss, W. (2012). Domestic Violence in Mexico: Perspectives of Mexican Counselors. Journal of Comparative Family Studies, 43(5), 751-772.

Parada, L. (1993). El concepto de familia. Patrones de distribución de ingreso. En P. Bedolla, O. Bustos, G. Delgado, B. García, \& L. Parada. Estudios de género y feminismo II (pp. 1-432). D.F., México: Fontamara.

Peña, F., Zamorano, B., Hernández, G., Hernández, M., Vargas, J., \& Parra, V. (2013). Violencia en el noviazgo en una muestra de jóvenes mexicanos. Revista Costarricense de Psicología, 32(1), 27-40.

Póo, A., \& Vizcarra, B. (2008). Violencia de pareja en jóvenes universitarios. Terapia Psicológica, 26(1), 8188.

Pueyo, A. (2009). La predicción de la violencia contra la pareja. En E. Echeburúa, J. Fernández-Montalvo, \& P. Corral (Eds.). Predicción del riesgo de bomicidio y de violencia grave en la relación de pareja. Instrumentos de evaluación del riesgo y adopción de medidas de protección (pp. 1-163). Valencia, España: Diseñarte-Goaprint, s.l.

Rey-Anacona, C. A. (2009). Maltrato de tipo físico, psicológico, emocional, sexual y económico en el noviazgo: un estudio exploratorio. Acta colombiana de psicología, 12(2), 27-36.

Rey-Anacona, C. A., Mateus-Cubides, A., \& BayonaArévalo, P. (2010). Malos tratos ejercidos por adolescents durante el noviazgo: diferencias pro sexo. Revista Mexicana de Psicología, 27(2), 169-181.

Rey-Anacona. C. A. (2013). Prevalencia y tipos de maltrato en el noviazgo en adolescentes y adultos jóvenes. Terapia psicológica, 31(2), 143-154
Rivera, S., Díaz-Loving, R., \& García, M. (2008). Etnopsicología del amor y el poder. En R. DíazLoving (Ed.). Etnopsicología mexicana. Siguiendo la buella teórica y empirica de Díaz-Guerrero (pp. 180-210). México: Trillas.

Rivera-Rivera, L., Allen-Leigh, B., Rodríguez-Ortega, G., Chávez-Ayala, R. \& Lazcano-Ponce, E., (2007). Prevalence and correlates of adolescent dating violence: baseline study of a cohort of 7960 male and female Mexican public school students. Preventive Medicine, 44(6), 477-484. doi:10.1016/j.ypmed.2007.02.020

Rojas-Solís, J. L. (2011a). Transformaciones socioculturales y aspectos de género: algunas implicaciones para el estudio de violencia en pareja. Revista Electrónica de Psicología Iztacala, 14(3), 252-272.

Rojas-Solís, J. L. (2011b). Violencia de pareja en universitarios españoles: resultados preliminares de un estudio exploratorio. International Journal of Developmental and Educational Psychology, 5(1), 571-581.

Rojas-Solís, J. L. (2013a). Violencia en el noviazgo y sociedad mexicana posmoderna. Algunos apuntes sobre la figura del agresor y las agresiones bidireccionales. Uaricha, 10(22), 1-19.

Rojas-Solís, J. L. (2013b). Violencia en el Noviazgo de Universitarios en México: Una Revisión. Revista Internacional de Psicología, 12(2), 1-31.

Rojas-Solís, J. L. (2013c). Violencia en el noviazgo de adolescentes mexicanos: una revisión. Revista de Educación y Desarrollo, (27), 49-58.

Rojas-Solís, J. L., \& Carpintero, E. (2011). Sexismo y agresiones físicas, sexuales y verbales.emocionales, en relaciones de noviazgo de estudiantes universitarios. Electronic Tournal of Research in Educational Psychology, 9(5), 541-564.

Romero, M. (2007). Violencia de género en las relaciones de pareja. Un estudio de caso. (Documento en sitio web) Recuperado de http://www.cubaenergia.cu/ genero/teoria/t48.pdf

Rubio-Garay, F., López-González, M. Á., Ángel, L., \& Sánchez-Elvira-Paniagua, Á. (2012). Direccionalidad 
y expresión de la violencia en las relaciones de noviazgo de los jóvenes. Acción Psicológica, 9(1), 61-70.

Ruiz, I. (2007). Violencia contra la mujer y salud. (Documento en sitio web) Recuperado de http:// www.msc.es/organizacion/sns/planCalidadSNS/ pdf/equidad/04modulo_03.pdf

Ruiz, Y. (2008). La violencia contra la mujer en la sociedad actual: análisis y propuestas de prevención. Recuperado de http://www.uji.es/bin/publ/edicions/ jfi13/18.pdf

Saewyc, E., Brown, D., Plane, M., Mundt, M., Zakletskaia, L., Wiegelc, J., \& Fleming, M. (2009). Gender Differences in Violence Exposure Among University Students Attending Campus Health Clinics in the United States and Canada. Journal of Adolescent Health, 45, 587-594. doi:10.1016/j. jadohealth.2009.03.024

Salazar, D., \& Vinet, E. (2011). Mediación familiar y volencia de pareja. Revista de Derecho, 24(1), 9-30.

Saldívar, G., Ramos, L., \& Romero, M. (2008). ¿Qué es la coreción sexual? significado, tácticas e interpretación en jóvenes universitarios de la cuidad de México. Salud Mental, 31(1), 45-51.

Saldivia, C. (2011). Representaciones sociales de la violencia en el pololeo: un estudio preliminar en estudiantes universitarios. Educación y Humanidades, 1(2), 83-99.

Sánchez, S. (2009). Estudio longitudinal del impacto de la violencia de pareja sobre la salud fisica y el sistema inmune de las mujeres. (Tesis doctoral, Universitat de València). Recuperado de http://www.tdx.cat/ bitstream/handle/10803/10204/sanchez.pdf;jse ssionid $=38756$ EDAFC9D1A74B3528358ADCE 7F34.tdx2? sequence $=1$

Sears, H., Byers, S., \& Price, L. (2007). The cooccurrence of adolescent boys' and girls' use of psychologically, physically, and sexually abusive behaviours in their dating relationships. Journal of Adolescence, 30, 487-504. doi:10.1016/j. adolescence.2006.05.002
Straus, H., Cerulli, C., McNutt, L., Rhodes, K. V., Conner, K. R., Kemball, R. S., Kaslow, N. \&Houry, D. (2009). Intimate Partner Violence and Functional Health Status: Associations with Severity, Danger, and Self-Advocacy Behaviors. Journal Of Women's Health, 18(5), 625-631. doi: 10.1089/jwh.2007.0521

Straus, M. (2006). Dominance and symmetry in partner violence by male and female university students in 32 Nations. En Intimate Violence Intervention (pp. 1-32). New York: University of Haifa and New York University.

Straus, M., \& Douglas, E. (2004). A short form of the Revised Conflicto Tactics Scales, and typologies for severety and mutuality. Violence and Victims, 19(5), 507-520.

Straus, M., \& Gozjolko, K. (2007). Intimate terrorism and injury of dating partners by male and female university students. Stockholm Criminology Symposium, (págs. 1-45). Stockholm, Sweden. Recuperado de http://pubpages. unh.edu/ mas2/CTS46X6.pdf

Straus, M., \& Mickey, E. (2012). Reliability, validity, and prevalence of partner violence measured by the conflict tactics scales in male-dominant nations. Aggression and Violent Behavior, 17, 463-474. doi:10.1016/j. avb.2012.06.004

Swahn, M., Simon, T., Hertz, M., Arias, I., Bossarte, R., Ross, J., Gross, L. A., Iachan, R., Hamburger, M. (2008). Linking Dating Violence, Peer Violence, and Suicidal. American Journal of Preventive Medicine Behaviors Among High-Riske Youth, 34(1), 30-38. doi: 10.1016/j.amepre.2007.09.020

Vara, A., \& Roa, Y. (2000). Negociación, ataque psicológico, ataque fisico, coerción sexualy daño fisico en parejas universitarias de Lima - Perú. Recuperado de C: \Users \Manoella \Documents \} DOCTORADO \Primer Semestre \Tesis $\backslash$ Bibliografia $\backslash$ Negociacion y Violencia en Parejas Universitarias de Lima.htm

Vázquez, V. \& Castro, R. (2008). ¿Mi novio sería capaz de matarme? Violencia en el noviazgo entre adolescentesde la Universidad Autónoma Chapingo, México. Lationoam. cienc.soc.niñer juw , 6(2), 709-738.

Villafañe, S., Jiménez, M., Carrasquillo, D., \& Vázquez, R. (2012). Construcción y validación del Cuestionario de

Actualidades en Psicología, 29(118), 2015, 57-72 
experiencias de violencia en las relaciones de pareja y familia en estudiantes universitarios. Universitas Psychologica, 11(1), 207-215.

Vizcarra, M., \& Póo, A. (2011). Violencia de pareja en estudiantes universitarios del sur de Chile. Universitas Psychologica, 10(1), 89-98.
Wekerle, C., Leungb, E., Wall, A.-M., MacMilland, H., Boyled, M., Trocmee, N., \& Waechterb, R. (2009). The contribution of childhood emotional abuse to teen dating violence among child protective services-involved youth. Child Abuse \& Neglect, 33, 45-58. doi: 0.1016/j. chiabu.2008.12.006

Recibido: 9 de setiembre de 2014 Aceptado: 3 de mayo de 2015 


\section{Anexo 1}

\section{Recopilación de estudios sobre perpetración, victimización y violencia mutua en el noviazgo}

\begin{tabular}{|c|c|c|c|c|}
\hline Autor (año) & Muestra & Diseño & País & Fuente \\
\hline Mañas, et. al. (2012) & $\begin{array}{l}394 \text { universitarios } \\
\text { entre 11-29 años }\end{array}$ & $\begin{array}{l}\text { Transversal } \\
\text { Cuantitativo }\end{array}$ & España & Google Academic \\
\hline Saewyc, et. al. (2009) & $\begin{array}{l}2091 \text { universitarios de } \\
18 \text { o más años }\end{array}$ & $\begin{array}{l}\text { Transversal } \\
\text { Cuantitativo }\end{array}$ & EUA & $\begin{array}{c}\text { Journal of } \\
\text { Adolescent Health }\end{array}$ \\
\hline Wekerle, et. al. (2009) & 402 jóvenes & $\begin{array}{l}\text { Transversal } \\
\text { Cuantitativo }\end{array}$ & Canadá & Child Abuse \& Neglect \\
\hline Murray, et. al. (2008) & $\begin{array}{l}1777 \text { universitarios de } \\
18 \text { o más años }\end{array}$ & $\begin{array}{l}\text { Transversal } \\
\text { Cuantitativo }\end{array}$ & EUA & $\begin{array}{l}\text { Journal of College } \\
\text { Counseling }\end{array}$ \\
\hline Swahn, et. al. (2008) & $\begin{array}{l}4131 \text { universitarios de } \\
18 \text { o más años }\end{array}$ & $\begin{array}{l}\text { Transversal } \\
\text { Cuantitativo }\end{array}$ & EUA & $\begin{array}{l}\text { American Journal of } \\
\text { Preventive Medicine }\end{array}$ \\
\hline Villafañe, et. al. (2012) & $\begin{array}{l}267 \text { universitarios entre } \\
16 \text { a } 20 \text { años }\end{array}$ & $\begin{array}{l}\text { Transversal } \\
\text { Cuantitativo }\end{array}$ & $\begin{array}{l}\text { Puerto } \\
\text { Rico }\end{array}$ & $\begin{array}{l}\text { Universitas } \\
\text { Psychologica }\end{array}$ \\
\hline Straus, et. al. (2009) & $\begin{array}{l}2737 \text { jóvenes } \\
\text { entre } 18 \text { a } 55 \text { años }\end{array}$ & $\begin{array}{l}\text { Transversal } \\
\text { Cuantitativo }\end{array}$ & EUA & $\begin{array}{c}\text { Journal of } \\
\text { Women's Health }\end{array}$ \\
\hline Oliva, et. al. (2012) & $\begin{array}{l}1988 \text { universitarios } \\
\text { entre } 19 \text { a } 49 \text { años }\end{array}$ & $\begin{array}{l}\text { Transversal } \\
\text { Cuantitativo }\end{array}$ & México & Revista Psicología.com \\
\hline $\begin{array}{l}\text { Rey-Anacona, } \\
\text { et. al. (2010). }\end{array}$ & $\begin{array}{l}562 \text { jóvenes } \\
\text { entre } 15 \text { a } 20 \text { años }\end{array}$ & $\begin{array}{l}\text { Transversal } \\
\text { Cuantitativo }\end{array}$ & Colombia & $\begin{array}{l}\text { Revista Mexicana } \\
\text { de Psicología }\end{array}$ \\
\hline $\begin{array}{l}\text { Muñoz-Rivas, } \\
\text { et. al. (2009). }\end{array}$ & $\begin{array}{c}4052 \text { jóvenes } \\
\text { entre } 16 \text { a } 26 \text { años }\end{array}$ & $\begin{array}{l}\text { Transversal } \\
\text { Cuantitativo }\end{array}$ & España & Psicothema \\
\hline Vara \& Roa (2000) & 713 estudiantes de pregrado & $\begin{array}{l}\text { Transversal } \\
\text { Cuantitativo }\end{array}$ & Perú & Google Academic \\
\hline Rey-Anacona (2013) & $\begin{array}{l}772 \text { jóvenes } \\
\text { entre } 15 \text { a } 35 \text { años }\end{array}$ & $\begin{array}{l}\text { Transversal } \\
\text { Cuantitativo }\end{array}$ & Colombia & Terapia Psicológica \\
\hline Gámez, et. al. (2011) & 13877 universitarios & $\begin{array}{l}\text { Transversal } \\
\text { Cuantitativo }\end{array}$ & Multipaís & Deviant Behavior \\
\hline Corral (2009) & $\begin{array}{l}1081 \text { universitarios } \\
\text { entre } 18 \text { a } 31 \text { años }\end{array}$ & $\begin{array}{l}\text { Transversal } \\
\text { Cuantitativo }\end{array}$ & España & $\begin{array}{c}\text { Psicopatología Clínica, } \\
\text { Legal y Forense }\end{array}$ \\
\hline Archer (2000) & 119 estudios & Metaanálisis & Multipaís & Psychological Bulletin \\
\hline Archer (2002) & 58 estudios & Metaanálisis & Multipaís & $\begin{array}{l}\text { Aggression and } \\
\text { Violent Behavior }\end{array}$ \\
\hline $\begin{array}{l}\text { Rivera-Rivera, } \\
\text { et. al. (2007) }\end{array}$ & $\begin{array}{c}7960 \text { jóvenes } \\
\text { entre 11-24 años }\end{array}$ & $\begin{array}{l}\text { Transversal } \\
\text { Cuantitativo }\end{array}$ & México & Preventive Medicine \\
\hline $\begin{array}{l}\text { Muñoz-Rivas, } \\
\text { et. al.. (2007). }\end{array}$ & $\begin{array}{l}2416 \text { estudiantes } \\
\text { entre } 16-20 \text { años }\end{array}$ & $\begin{array}{l}\text { Transversal } \\
\text { Cuantitativo }\end{array}$ & España & $\begin{array}{c}\text { Journal of } \\
\text { Adolescent Health }\end{array}$ \\
\hline
\end{tabular}




\begin{tabular}{|c|c|c|c|c|}
\hline Autor (año) & Muestra & Diseño & País & Fuente \\
\hline $\begin{array}{l}\text { Desmarais, et. } \\
\text { al. (2012a) }\end{array}$ & $\begin{array}{l}249 \text { artículos (2000-2010). } \\
\text { Criterios: victimización de } \\
\text { violencia física, relación } \\
\text { heterosexual, y muestra de } \\
\text { adolescents y adultos jóvenes. }\end{array}$ & $\begin{array}{l}\text { Revisión de } \\
\text { literatura }\end{array}$ & Multipaís & Partner Abuse \\
\hline $\begin{array}{l}\text { Desmarais, et. } \\
\text { al. }(2012 b)\end{array}$ & $\begin{array}{l}111 \text { artículos }(2000-2010) \text {. } \\
\text { Criterios: perpetración de } \\
\text { violencia física, relación } \\
\text { heterosexual y edad de } 13 \text { o más }\end{array}$ & $\begin{array}{l}\text { Revisión de } \\
\text { literatura }\end{array}$ & Multipaís & Partner Abuse \\
\hline O’Leary, et. al. (2008) & $\begin{array}{l}2363 \text { estudiantes } \\
\text { entre } 15-18 \text { años }\end{array}$ & $\begin{array}{l}\text { Transversal } \\
\text { Cuantitativo }\end{array}$ & EUA & $\begin{array}{c}\text { Journal of } \\
\text { Adolescent Health }\end{array}$ \\
\hline Álvarez (2012) & $\begin{array}{l}\text { Más de } 400 \text { estudios. } \\
\text { Actos perpetrados y sufridos por } \\
\text { ambos miembros de la pareja }\end{array}$ & Metaanálisis & Multipaís & Academia.edu \\
\hline Comezaña (2006) & Estudiantes universitarios & $\begin{array}{l}\text { Transversal } \\
\text { Cuantitativo }\end{array}$ & Perú & Tesis no publicada \\
\hline $\begin{array}{l}\text { Lehrer, Lehrer \& } \\
\text { Zhenxiang (2009) }\end{array}$ & $\begin{array}{c}950 \text { universitarios: } \\
484 \text { mujeres y } 466 \text { hombres }\end{array}$ & $\begin{array}{l}\text { Transversal } \\
\text { Cuantitativo }\end{array}$ & Chile & $\begin{array}{l}\text { International of } \\
\text { Injury Control and } \\
\text { Safety Promotion }\end{array}$ \\
\hline $\begin{array}{l}\text { Instituto Mexicano de } \\
\text { la Juventud (2008) }\end{array}$ & $\begin{array}{c}7 \text { millones } 278 \text { mil } 236 \text { jóvenes } \\
\text { entre } 15-24 \text { años }\end{array}$ & $\begin{array}{l}\text { Transversal } \\
\text { Cuantitativo }\end{array}$ & México & Google Academic \\
\hline Peña (2013) & $\begin{array}{l}140 \text { jóvenes } \\
\text { entre 19-25 años }\end{array}$ & $\begin{array}{l}\text { Transversal } \\
\text { Cuantitativo }\end{array}$ & México & $\begin{array}{l}\text { Revista Costarricense } \\
\text { de Psicología }\end{array}$ \\
\hline Rojas-Solís (2013a) & $\begin{array}{l}15 \text { estudios (2003-2013) con } \\
\text { jóvenes entre 12-21 años }\end{array}$ & Metaanálisis & México & $\begin{array}{c}\text { Revista de Educación } \\
\text { y Desarrollo }\end{array}$ \\
\hline Rojas-Solís (2013b) & $\begin{array}{l}20 \text { estudios de jóvenes } \\
\text { entre 18-25 años }\end{array}$ & $\begin{array}{l}\text { Revision de } \\
\text { la literatura }\end{array}$ & Multipaís & $\begin{array}{l}\text { Revista Internacional } \\
\text { de Psicología }\end{array}$ \\
\hline Giordano, et. al. (2010). & 956 jóvenes Preuniversitarios & $\begin{array}{l}\text { Transversal } \\
\text { Cuantitativo }\end{array}$ & EUA & Social Science Research \\
\hline Leal, et. al. (2011). & 360 jóvenes entre $14-18$ años & $\begin{array}{l}\text { Transversal } \\
\text { Cuantitativo }\end{array}$ & Chile & $\begin{array}{l}\text { Revista Infancia } \\
\text { y Educación }\end{array}$ \\
\hline $\begin{array}{c}\text { Medeiros \& } \\
\text { Straus (2006) }\end{array}$ & $\begin{array}{c}854 \text { universitarios: } \\
312 \text { hombres } 542 \text { mujeres }\end{array}$ & $\begin{array}{l}\text { Transversal } \\
\text { Cuantitativo }\end{array}$ & EUA & pubpages.unh.ed \\
\hline Straus \& Mickey (2012) & $\begin{array}{l}14252 \text { universitarios con } \\
\text { media de } 23 \text { años de edad }\end{array}$ & $\begin{array}{l}\text { Transversal } \\
\text { Cuantitativo }\end{array}$ & Multipaís & $\begin{array}{l}\text { Agression and } \\
\text { Violent Behavior }\end{array}$ \\
\hline Straus (2006) & 13601 universitarios & $\begin{array}{l}\text { Transversal } \\
\text { Cuantitativo }\end{array}$ & Multipaís & $\begin{array}{l}\text { Conferencia en } \\
\text { pubpages.unh.ed }\end{array}$ \\
\hline Arias, et. al. (2010) & $\begin{array}{l}250 \text { universitarios } \\
\text { entre } 15-25 \text { años }\end{array}$ & $\begin{array}{l}\text { Transversal } \\
\text { Cuantitativo }\end{array}$ & México & Google Academic \\
\hline $\begin{array}{l}\text { Straus \& Douglas } \\
\qquad(2004)\end{array}$ & $\begin{array}{c}1157 \text { universitarios: } \\
347 \text { hombres y } 810 \text { mujeres }\end{array}$ & $\begin{array}{l}\text { Transversal } \\
\text { Cuantitativo }\end{array}$ & EUA & pubpages.unh.ed \\
\hline
\end{tabular}




\begin{tabular}{|c|c|c|c|c|}
\hline Autor (año) & Muestra & Diseño & País & Fuente \\
\hline $\begin{array}{l}\text { Muñoz-Rivas, Andreu, } \\
\text { et. al. (2007) }\end{array}$ & $\begin{array}{l}5355 \text { jóvenes } \\
\text { entre 16-26 años }\end{array}$ & $\begin{array}{l}\text { Transversal } \\
\text { Cuantitativo }\end{array}$ & España & Psicothema \\
\hline $\begin{array}{l}\text { Straus \& Gozjolko } \\
\quad(2007)\end{array}$ & $\begin{array}{c}13877 \text { universitarios: } \\
3886 \text { hombres y } 9991 \text { mujeres }\end{array}$ & $\begin{array}{r}\text { Transversal } \\
\text { Cuantitativo }\end{array}$ & Multipaís & pubpages.unh.ed \\
\hline $\begin{array}{l}\text { Fernández-Fuertes } \\
\text { \& Fuertes (2010) }\end{array}$ & $\begin{array}{l}567 \text { jóvenes: } 236 \text { hombres y } \\
331 \text { mujeres entre } 15-19 \text { años }\end{array}$ & $\begin{array}{l}\text { Transversal } \\
\text { Cuantitativo }\end{array}$ & España & $\begin{array}{l}\text { Artículo en Child } \\
\text { Abuse \& Neglect }\end{array}$ \\
\hline Romero (2007) & $\begin{array}{l}\text { Dos estudios de caso: relaciones } \\
\text { de pareja jóvenes 21-24 años }\end{array}$ & $\begin{array}{l}\text { Transversal } \\
\text { Cualitativo }\end{array}$ & Cuba & cubaenergia.cu \\
\hline Rojas-Solís (2011b) & $\begin{array}{l}174 \text { universitarios: } 47 \text { hombres } \\
\text { y } 127 \text { mujeres entre } 18-21 \text { años }\end{array}$ & $\begin{array}{l}\text { Transversal } \\
\text { Cuantitativo }\end{array}$ & España & $\begin{array}{l}\text { International Journal } \\
\text { of Developmental and } \\
\text { Educational Psychology }\end{array}$ \\
\hline $\begin{array}{l}\text { Rubio-Garay, } \\
\text { et. al. (2012) }\end{array}$ & $\begin{array}{l}69 \text { jóvenes: } 34 \text { hombres y } 35 \\
\text { mujeres entre 16-27 años }\end{array}$ & $\begin{array}{l}\text { Transversal } \\
\text { Cuantitativo }\end{array}$ & España & Acción Psicológica \\
\hline $\begin{array}{l}\text { Graña, Rodríguez } \\
\text { \& Peña (2009) }\end{array}$ & 1897 de más de 18 años & $\begin{array}{l}\text { Transversal } \\
\text { Cuantitativo }\end{array}$ & España & $\begin{array}{l}\text { Psicopatología Clínica, } \\
\text { Legal y Forense }\end{array}$ \\
\hline $\begin{array}{l}\text { Fernández-Fuertes, } \\
\text { Fuertes \& Pulido (2006) }\end{array}$ & $\begin{array}{l}572 \text { jóvenes } \\
\text { entre 15-19 años }\end{array}$ & $\begin{array}{l}\text { Transversal } \\
\text { Cuantitativo }\end{array}$ & España & $\begin{array}{c}\text { International Journal } \\
\text { of Clinical of Health } \\
\text { Psychology }\end{array}$ \\
\hline $\begin{array}{l}\text { Sears, Byers \& } \\
\text { Price (2007) }\end{array}$ & $\begin{array}{l}633 \text { adolescentes: } 309 \\
\text { mujeres y } 324 \text { hombres }\end{array}$ & $\begin{array}{l}\text { Transversal } \\
\text { Cuantitativo }\end{array}$ & Canadá & Journal of Adolescence \\
\hline Moral \& López (2013b) & $\begin{array}{l}400 \text { participantes: } 223 \\
\text { mujeres y } 177 \text { hombres }\end{array}$ & $\begin{array}{r}\text { Transversal } \\
\text { Cuantitativo }\end{array}$ & México & Psicogente \\
\hline $\begin{array}{c}\text { Rojas-Solís \& } \\
\text { Carpintero (2011) }\end{array}$ & $\begin{array}{l}453 \text { universitarios } \\
\text { entre } 18-36 \text { años }\end{array}$ & $\begin{array}{l}\text { Transversal } \\
\text { Cuantitativo }\end{array}$ & España & $\begin{array}{c}\text { Electronic Journal of } \\
\text { Research in Educational } \\
\text { Psychology }\end{array}$ \\
\hline
\end{tabular}

